\title{
X-Ray Measurements of the Particle Acceleration Properties at Inward Shocks in Cassiopeia A
}

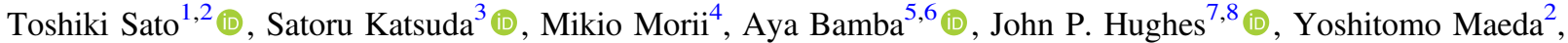 \\ Manabu Ishida ${ }^{2}$, and Federico Fraschetti ${ }^{9}$ (1) \\ ${ }^{1}$ Department of Physics, Tokyo Metropolitan University, 1-1 Minami-Osawa, Hachioji, Tokyo 192-0397, Japan; toshiki@astro.isas.jaxa.jp \\ ${ }^{2}$ Department of High Energy Astrophysics, Institute of Space and Astronautical Science (ISAS), Japan Aerospace Exploration Agency (JAXA), \\ 3-1-1 Yoshinodai, Sagamihara, 229-8510, Japan \\ ${ }^{3}$ Department of Physics, Faculty of Science \& Engineering, Chuo University, 1-13-27 Kasuga, Bunkyo, Tokyo 112-8551, Japan \\ ${ }^{4}$ The Institute of Statistical Mathematics, 10-3 Midori-cho, Tachikawa, Tokyo 190-8562, Japan \\ ${ }^{5}$ Department of Physics, The University of Tokyo, 7-3-1 Hongo, Bunkyo-ku, Tokyo 113-0033, Japan \\ ${ }^{6}$ Research Center for the Early Universe, School of Science, The University of Tokyo, 7-3-1 Hongo, Bunkyo-ku, Tokyo 113-0033, Japan \\ Department of Physics and Astronomy, Rutgers University, 136 Frelinghuysen Road, Piscataway, NJ 08854-8019, USA \\ ${ }^{8}$ Center for Computational Astrophysics, Flatiron Institute, 162 Fifth Avenue, New York, NY 10010, USA \\ ${ }^{9}$ Department of Planetary Sciences and Astronomy, University of Arizona, Tucson, AZ, 85721, USA \\ Received 2017 October 17; revised 2017 December 5; accepted 2017 December 5; published 2018 January 22
}

\begin{abstract}
We present new evidence that the bright nonthermal X-ray emission features in the interior of the Cassiopeia A supernova remnant are caused by inward-moving shocks, based on Chandra and NuSTAR observations. Several bright inward-moving filaments were identified using monitoring data taken by Chandra in 2000-2014. These inward-moving shock locations are nearly coincident with hard X-ray (15-40 keV) hot spots seen by NuSTAR. From proper-motion measurements, the transverse velocities were estimated to be in the range of $\sim 2100-3800 \mathrm{~km} \mathrm{~s}^{-1}$ for a distance of $3.4 \mathrm{kpc}$. The shock velocities in the frame of the expanding ejecta reach values of $\sim 5100-8700 \mathrm{~km} \mathrm{~s}{ }^{-1}$, which is slightly higher than the typical speed of the forward shock. Additionally, we find flux variations (both increasing and decreasing) on timescales of a few years in some of the inward-moving shock filaments. The rapid variability timescales are consistent with an amplified magnetic field of $B \sim 0.5-1 \mathrm{mG}$. The high speed and low photon cut-off energy of the inward-moving shocks are shown to imply a particle diffusion coefficient that departs from the Bohm regime $\left(k_{0}=D_{0} / D_{0, \mathrm{Bohm}} \sim 3-8\right)$ for the few simple physical configurations we consider in this study. The maximum electron energy at these shocks is estimated to be $\sim 8-11 \mathrm{TeV}$, which is smaller than the values of 15-34 TeV that were inferred for the forward shock. Cassiopeia A is dynamically too young for its reverse shock to appear to be moving inward in the observer frame. We propose instead that the inward-moving shocks are a consequence of the forward shock encountering a density jump of $\gtrsim 5-8$ in the surrounding material.
\end{abstract}

Key words: acceleration of particles - ISM: supernova remnants - supernovae: individual (Cassiopeia A) X-rays: ISM

\section{Introduction}

Shock waves in young supernova remnants (SNRs) are excellent astrophysical laboratories for understanding the process of particle acceleration. An important observational advance was the imaging of synchrotron X-ray-emitting rims in SN1006 (Koyama et al. 1995), which strengthened arguments for the shock acceleration of electrons to $\mathrm{TeV}$ energies in the forward shocks of young SNRs. More recently, $\mathrm{GeV}$ and $\mathrm{TeV} \gamma$-ray spectral observations have been shown to be well described by the decay of pions produced by energetic protons interacting with ambient gas (e.g., Ackermann et al. 2013), providing strong evidence for proton acceleration in SNRs. From these studies, as well as from several others using a variety of different techniques, it is now widely accepted that electron and proton acceleration occurs in SNRs. Diffusive shock acceleration (DSA; Bell 1978; Blandford \& Eichler 1987) in the forward shocks of remnants is the leading candidate for the process and can account for the majority of Galactic cosmic rays. On the other hand, observational evidence for particle acceleration at SNR reverse shocks, which propagate inward through the expanding ejecta, has not been clearly demonstrated, although there have been some theoretical studies (e.g., Ellison et al. 2005; Telezhinsky et al. 2012, 2013).
With an age of $\sim 350$ years (Fesen et al. 2006), Cassiopeia A is one of the most well-studied young Galactic SNRs. Nonthermal X-ray emission have been detected from both the outer rim and interior positions (e.g., Hughes et al. 2000; Vink \& Laming 2003; Bamba et al. 2005; Helder \& Vink 2008; Maeda et al. 2009; Grefenstette et al. 2015). In particular, the brightest nonthermal X-ray emission comes from the western limb that is slightly interior to the forward-shock position and is also strongly associated with $\mathrm{TeV} \gamma$-ray emission (e.g., Aharonian et al. 2001; Albert et al. 2007; Maeda et al. 2009; Acciari et al. 2010; Grefenstette et al. 2015). Those features naturally suggest an efficient production of accelerated particles in this region; however, the origin of the energetic electrons in the interior of the remnant remains a mystery.

Radio and X-ray observations of Cassiopeia A have found that some features are moving back toward the expansion center (Anderson \& Rudnick 1995; Keohane et al. 1996; DeLaney et al. 2004); we refer to these as "inward shocks." The inward shocks are concentrated in the south and west between azimuths of $170^{\circ}-300^{\circ}$. Helder \& Vink (2008) have suggested that the bright nonthermal X-rays at the reverse-shock region could be due to the locally higher reverse-shock velocity $\left(v_{s} \sim 6,000 \mathrm{~km} \mathrm{~s}^{-1}\right)$ of the inward-moving shock filaments. It was recognized even earlier that such high reverse-shock velocities or inward-moving 
filaments required a more complex structure for the circumstellar medium in the west. For example, Keohane et al. (1996) argued for a physical connection with a local molecular cloud, based on strong radio and X-ray absorbing structures seen near the inwardmoving shocks (see also Sanders 2006 and Hwang \& Laming 2012 for the X-ray absorbing structures). Recent millimeter observations have additionally suggested an interaction between the shock front and a nearby molecular cloud (Kilpatrick et al. 2014). Such a high density cloud would also be consistent with the pion-decay scenario for the $\gamma$-ray emission from Cassiopeia A. Finally, a shock-cloud interaction is considered to have a connection with the nonthermal X-rays (Sano et al. 2010, 2013, 2015; Inoue et al. 2012).

Although these various nonthermal features appear to be related, a unified picture for the western reverse-shock region of Cassiopeia A has yet to be established. As a step in further understanding the particle acceleration properties in this exceptional region of the remnant, here we report new X-ray kinematic and intensity properties for bright inward-moving filaments. Using long-term monitoring data from Chandra, we determine the proper motion and temporal flux variation for a set of six bright nonthermal filaments. We then combine this with results from hard X-ray measurements by NuSTAR to determine the physical properties (e.g., magnetic field, diffusion coefficient, maximum electron energy, and acceleration and cooling timescales) of the particle acceleration process in these shocks.

\section{Observation and Data Reduction}

\subsection{Chandra}

The Advanced CCD Imaging Spectrometer (ACIS-S) of Chandra has observed Cassiopeia A several times since its launch in 1999 (e.g., Hwang et al. 2000, 2004; Patnaude et al. 2011; Hwang \& Laming 2012; Patnaude \& Fesen 2014). The data used in our analysis are listed in Table 1 . We reprocessed the event files (from level 1 to level 2) to remove pixel randomization and to correct for CCD charge transfer efficiencies using CIAO (Fruscione et al. 2006) version 4.6 and CalDB 4.6.3. The bad grades were filtered out and good time intervals were reserved.

In order to align the images, we used the Central Compact Object (CCO) as a fiducial source. Because of lack of other certain fiducial sources around Cassiopeia A, we registered only the $\mathrm{CCO}$ for the alignment. First, we determined the $\mathrm{CCO}$ position using wavdetect implemented in CIAO ${ }^{10}$ Next, we shift values between two ObsIDs that were computed with the wCS_match script. Using these values, we finally updated the aspect solutions of the event files using wcs_update. We here used the ObsID 4638 as the reference of the alignment. The shift values are summarized in Table 1. In the following Section 3.2, we assumed official systematic errors of $\sim 0 .{ }^{\prime \prime} 5^{11}$ for the positional accuracy, although we aligned the images. Our image alignment using the $\mathrm{CCO}$ could reduce the uncertainties. However, there would still be other positional uncertainties (e.g., roll angle, proper motion of the $\mathrm{CCO}$, etc.). For example, Fesen et al. (2006) have estimated a transverse velocity of $\simeq 350 \mathrm{~km} \mathrm{~s}^{-1}$ for the CCO. This corresponds to a shift of $\sim 0$ "! 3 ( $<1$ pixel) for

\footnotetext{
${ }^{10}$ http://cxc.harvard.edu/ciao/

11 See http://cxc.harvard.edu/cal/ASPECT/celmon/ for the Chandra absolute astrometric accuracy.
}

Table 1

Chandra Observations

\begin{tabular}{lcccc}
\hline \hline ObsID. & $\begin{array}{c}\text { Date } \\
\text { YYYY MM DD }\end{array}$ & $\begin{array}{c}\text { Exp. } \\
(\mathrm{ks})\end{array}$ & $\begin{array}{c}\text { Angle } \\
(\mathrm{deg})\end{array}$ & $\begin{array}{c}\text { Shift Values } \\
(\Delta x, \Delta y)^{\mathrm{a}}\end{array}$ \\
\hline 114 & 2000 Jan 30 & 49.9 & 323.4 & $(-0.09,+0.34)$ \\
\hline 1952 & 2002 Feb 06 & 49.6 & 323.4 & $(-0.36,+0.38)$ \\
\hline 4634 & 2004 Apr 28 & 148.6 & 59.2 & $(+0.15,-0.20)$ \\
4635 & 2004 May 01 & 135.0 & 59.2 & $(-0.05,-0.27)$ \\
4636 & 2004 Apr 20 & 143.5 & 49.8 & $(-0.09,-0.21)$ \\
4637 & 2004 Apr 22 & 163.5 & 49.8 & $(-0.04,-0.22)$ \\
4638 & 2004 Apr 14 & 164.5 & 40.3 & $($ reference) \\
4639 & 2004 Apr 25 & 79.0 & 49.8 & $(-0.04,-0.35)$ \\
5196 & 2004 Feb 08 & 49.5 & 325.5 & $(+0.07,+0.03)$ \\
5319 & 2004 Apr 18 & 42.3 & 49.8 & $(-0.08,+0.08)$ \\
5320 & 2004 May 05 & 54.4 & 65.1 & $(+0.03,-0.41)$ \\
\hline 9117 & 2007 Dec 05 & 24.8 & 278.1 & $(-0.01,+0.87)$ \\
9773 & 2007 Dec 08 & 24.8 & 278.1 & $(-0.19,+0.89)$ \\
\hline 10935 & 2009 Nov 02 & 23.3 & 239.7 & $(-0.53,+0.53)$ \\
12020 & 2009 Nov 03 & 22.4 & 239.7 & $(-0.50,+0.32)$ \\
\hline 10936 & 2010 Oct 31 & 32.2 & 236.5 & $(-0.44,-0.11)$ \\
13177 & 2010 Nov 02 & 17.2 & 236.5 & $(+0.00,-0.02)$ \\
\hline 14229 & 2012 May 15 & 49.1 & 75.4 & $(-0.52,-0.60)$ \\
\hline 14480 & 2013 May 20 & 48.8 & 75.1 & $(-0.38,+0.63)$ \\
\hline 14481 & 2014 May 12 & 48.4 & 75.1 & $(+0.08,+0.50)$ \\
\hline & & & & \\
\hline
\end{tabular}

Note.

${ }^{a}$ Sky pixel value (1 pixel $=0$ ". 492).

$14 \mathrm{yr}$ at the distance of $3.4 \mathrm{kpc}$ (see Reed et al. 1995 for the distance), which is within the systematic errors.

\subsection{NUSTAR}

NUSTAR, which is composed of two co-aligned X-ray telescopes (focal plane module A (FPMA) and focal plane module $\mathrm{B}$ (FPMB)) that observe the sky in the energy range of 3-79 keV (Harrison et al. 2013), has observed Cassiopeia A with a total of $\sim 2.4 \mathrm{Ms}$ of exposure time during the first two years after the launch in 2012 (see Table 2). The first hard $\mathrm{X}$-ray imaging revealed a ${ }^{44} \mathrm{Ti}$ distribution and a morphology of $>15 \mathrm{keV}$ emission in Cassiopeia $\mathrm{A}$ for the first time (Grefenstette et al. 2014, 2015, 2017).

In order to compare the hard X-ray image (15-40 keV) with the Chandra image (4.2-6 keV), we analyzed all of the NUSTAR data. We reduced the data with the NUSTAR Data Analysis Software (NuSTARDAS) version 1.6.0 and the NUSTAR calibration database (CALDB) version 20170503 to produce images and exposure maps for each telescope. All of the images were merged using XIMAGE, taking into account the exposure maps (i.e., the unique time-dependent exposure and the vignetting function of the telescope) for each pointing. After the correction, we deconvolved the merged image with the on-axis point spread function (PSF) image of NuSTAR's telescope. We used arestore script in CIAO for the deconvolution. Following the method in Grefenstette et al. (2015), we chose to halt the deconvolution after 50 iterations with a Richardson-Lucy method, and we obtained almost the 
Table 2

NUSTAR Observations

\begin{tabular}{lcc}
\hline \hline ObsID. & $\begin{array}{c}\text { Date } \\
\text { YYYY MM DD }\end{array}$ & $\begin{array}{c}\text { Exp. } \\
(\mathrm{ks})\end{array}$ \\
\hline 40001019002 & 2012 Aug 18 & 294 \\
40021001002 & 2012 Aug 27 & 190 \\
40021001004 & 2012 Oct 07 & 29 \\
40021001005 & 2012 Oct 07 & 228 \\
40021002002 & 2012 Nov 27 & 288 \\
40021002006 & 2013 Mar 02 & 160 \\
40021002008 & 2013 Mar 05 & 226 \\
40021002010 & 2013 Mar 09 & 16 \\
40021003002 & 2013 May 28 & 13 \\
40021003003 & 2013 May 28 & 216 \\
40021011002 & 2013 Oct 30 & 246 \\
40021012002 & 2013 Nov 27 & 239 \\
40021015002 & 2013 Dec 21 & 86 \\
40021015003 & 2013 Nov 23 & 160 \\
\hline Total & & $\sim 2.4 \mathrm{Ms}$ \\
\hline
\end{tabular}

same image as the published image (see Figure 1). For the NuSTAR imaging, the default aspect solution was used.

\section{Analysis and Results}

\subsection{Soft and Hard X-Ray Imaging}

In Figure 1(a), we show the different between 2000 and 2014 generated from Chandra data. Here, we can see moving X-ray structures, as previously reported (e.g., DeLaney et al. 2004; Patnaude \& Fesen 2009). All of the forward-shock filaments that are located at the outer rim are moving outward. On the other hand, there are several filaments near the center and toward the west that are moving inward (such filaments are identified with green arrows in the figure). Those filaments are the same inward-moving features (inward shocks) as reported in DeLaney et al. (2004). In particular, almost all of the filaments toward the west seem to be inward shocks. By definition, the forward shock must always move to the outside of the remnant. Therefore, these inward shocks cannot be the forward shocks that were seen projected on the interior of the remnant. In addition, part of the eastern region shows a uniform dark color (see the broken ellipse region in Figure 1). The eastern region is known to have a large contribution from thermal X-rays with a flux decline, which is possibly due to adiabatic expansion (Sato et al. 2017). The dark eastern region in this figure is just another view of the prominent flux decay of the thermal emission.

In Figure 1(b), we show the 15-40 keV image with NuSTAR. As reported in previous studies (Willingale et al. 2002; Helder \& Vink 2008; Maeda et al. 2009; Grefenstette et al. 2015; Sato et al. 2017), most of the hard X-rays are located at the west and the center. Thus, we find a strong correlation between the inward-shock positions and the intensity peaks of the NUSTAR image.

In Figure 1(c), we compared the NUSTAR image with the soft X-ray images (4.2-6 keV; continuum emission and 6.54-6.92 keV; Fe-K emission) with Chandra. As reported in Sato et al. (2017), we can see a separation between the thermal X-ray distribution $(\mathrm{Fe}-\mathrm{K})$ and the hard X-ray distribution (which is spatially coincident with the inward-shock positions).
To clarify the motions of the shock filaments, we also compared images taken in 2004 (ObsID. 5320) and 2014 (ObsID. 14481) using a computer vision technique (optical flow). The dense optical-flow algorithm we use is by Gunnar Farnebäck (Farnebäck 2003), as implemented in OpenCV 3.2.0. ${ }^{12}$ This algorithm estimates displacement fields between two frames using quadratic polynomials to locally approximate the image flux. To reduce the noise, the estimate is made by averaging over local neighborhoods. Also, we here set statistical limits for the surface brightnesses. For the continuum and $\mathrm{Si}-\mathrm{K}$ images, we ignore pixels whose surface brightnesses are $<1 \times 10^{-7}$ counts $\mathrm{cm}^{-2} \mathrm{~s}^{-1}$ and $<3 \times 10^{-7}$ counts $\mathrm{cm}^{-2} \mathrm{~s}^{-1}$, respectively, which corresponds to $\lesssim 1$ counts with a $\sim 50 \mathrm{ksec}$ exposure time. Specifically, we used the calcOpticalFlowFarneback function with the following arguments: pyr_scale $=0.5$, which specifies the image scale to build pyramids for each image; levels $=3$; the number of pyramid layers; winsize $=15$; averaging window size; and poly_n $=5$, which is the size of the pixel neighborhood used for the polynomial approximation. As shown in Figure 2, we can visualize the motion of filaments (or small knots) as vector maps over the entire extent of the SNR. In the vector maps (Figures 2(a) and (b)), we plot only large vectors whose proper motions are larger than $\sim 0.05 \operatorname{arcsec} \mathrm{yr}^{-1}\left(\approx 800 \mathrm{~km} \mathrm{~s}^{-1}\right.$ at the distance of $3.4 \mathrm{kpc}$ ), and we found that the large motions are strongly associated with filamentary (or knotty) structures. Also, we can see clearly that large motions are not identified at low count-rate regions.

In the continuum band (Figures 2(a) and $\left(\mathrm{a}^{\prime}\right)$ ), the inward motions are concentrated in the interior. Indeed, the positions of inward-moving features (the blue spots in Figure $2\left(\mathrm{a}^{\prime}\right)$ ) are very similar to the locations of the hard X-ray spots in Figure 1(b), providing further support for a connection between them. On the other hand, almost all of the small structures in the $\mathrm{Si}-\mathrm{K}$ band are moving to the outside of the remnant (Figures 2(b) and $\left.\left(b^{\prime}\right)\right)$. These indicate a clear difference in the character of motion between the ejecta and the nonthermal emission components.

In this current study, we employ the optical-flow method to provide a largely qualitative view of the expansion of the thermal ejecta and nonthermal shock filaments in Cassiopeia A. A detailed quantitative study of the results is beyond the scope of this initial investigation, although in future work, we will determine the accuracy and robustness of optical-flow propermotion measurements for determining the global kinematics of young SNRs. In particular, combining comprehensive propermotion and radial velocity measurements will further improve our understanding of the 3D kinetics of young SNRs (e.g., DeLaney et al. 2010; Sato \& Hughes 2017a, 2017b; Williams et al. 2017).

\subsection{Proper-motion Measurements of the Inward-shock Filaments}

To measure the proper motions of the inward-shock filaments, we extracted images from the box regions in Figure 1 and fit them with an image model. The fitting code used for this analysis was originally developed for proper-motion measurements for knots in Kepler's SNR (Sato \& Hughes 2017b). Here, we used the 2004 image normalized by the image in each epoch as the model because the observation in 2004 has the longest exposure time,

\footnotetext{
${ }^{12}$ See http://opencv.org/ for more details.
} 

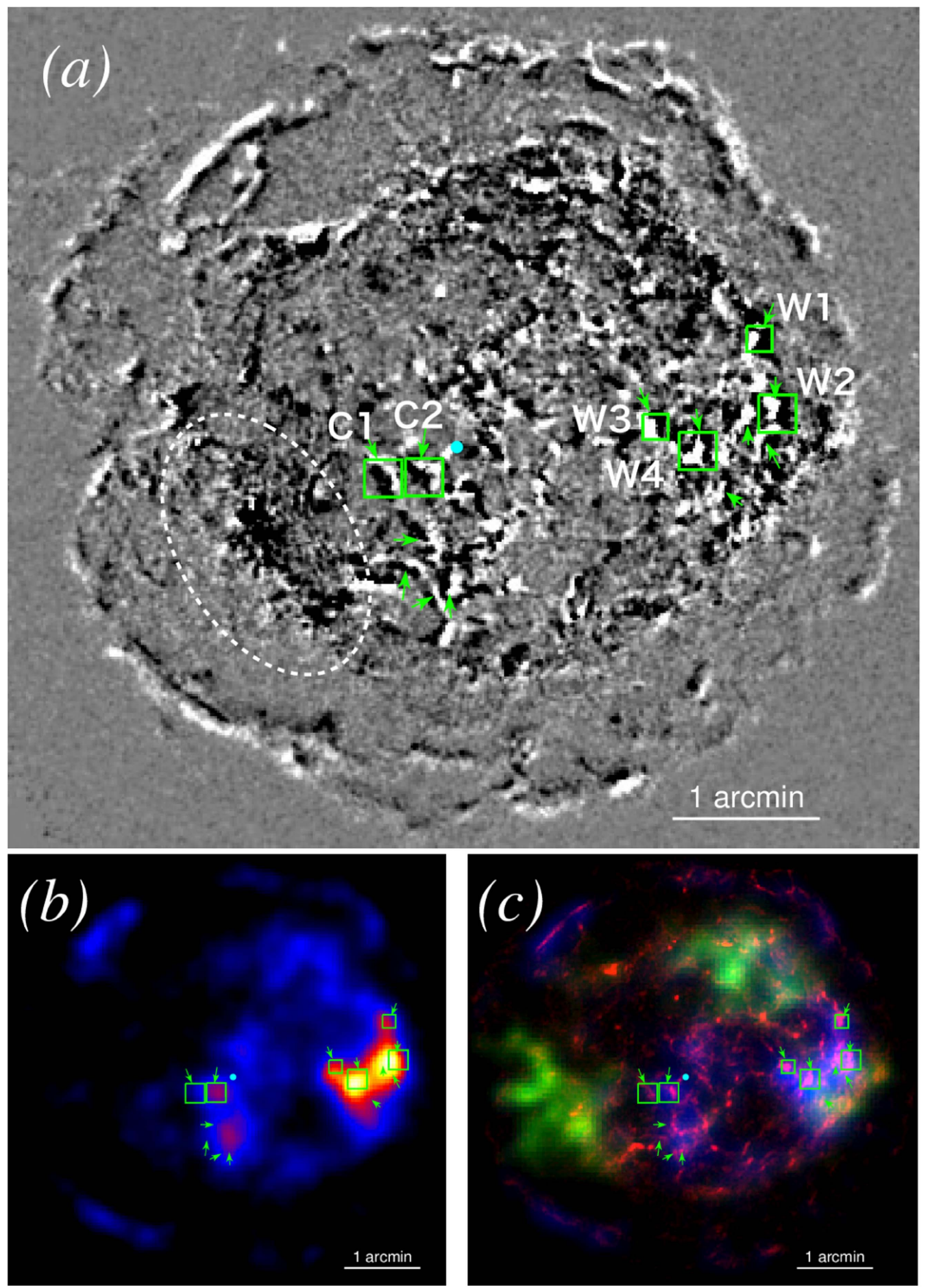

Figure 1. (a) Image difference in the $4.2-6 \mathrm{keV}$ band of Cassiopeia A between 2000 and 2014 with Chandra. Adjacent black and white features show transverse motions of X-ray structures. Green arrows and boxes show the inward-shock positions defined by eye and the regions that were used for the proper-motion measurements. The boxes are identified by name (e.g., C1, W1) The box sizes are $21 \times 21$ pixels $(1$ pixel $=0$ !! 492$)$ for W1 and W3, and $31 \times 31$ pixels for C1, C2, W2, and W4, respectively. (b) The NUSTAR image in 15-40 keV band. Green arrows and boxes show the same as shown in the panel (a). (c) Three-color image of Cassiopeia A. Red, green, and blue colors show the 4.2-6 keV image with Chandra, the 6.54-6.92 keV image (around Fe-K emission) with Chandra, and the 15-40 keV image with NuSTAR, respectively. The binning sizes for the 4.2-6 keV (Chandra), 6.54-6.92 keV (Chandra), and 15-40 keV (NuSTAR) images are $1 \times 1$ pixels $(0$ "! 492 × 0". 492),

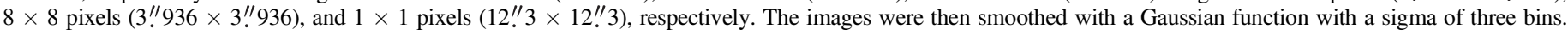
Green arrows and boxes show the same as shown in the panel (a). The cyan dot shows the position of the CCO.

which is about 20 times longer than that of the other observations (see Table 1). For obtaining the best-fit shifts, a maximum likelihood statistic for Poisson distributions was used (C-statistics;
Cash 1979), which minimizes

$$
C=-2 \ln P=-2 \Sigma_{i, j}\left(n_{i, j} \ln m_{i, j}-m_{i, j}-\ln n_{i, j} !\right)
$$




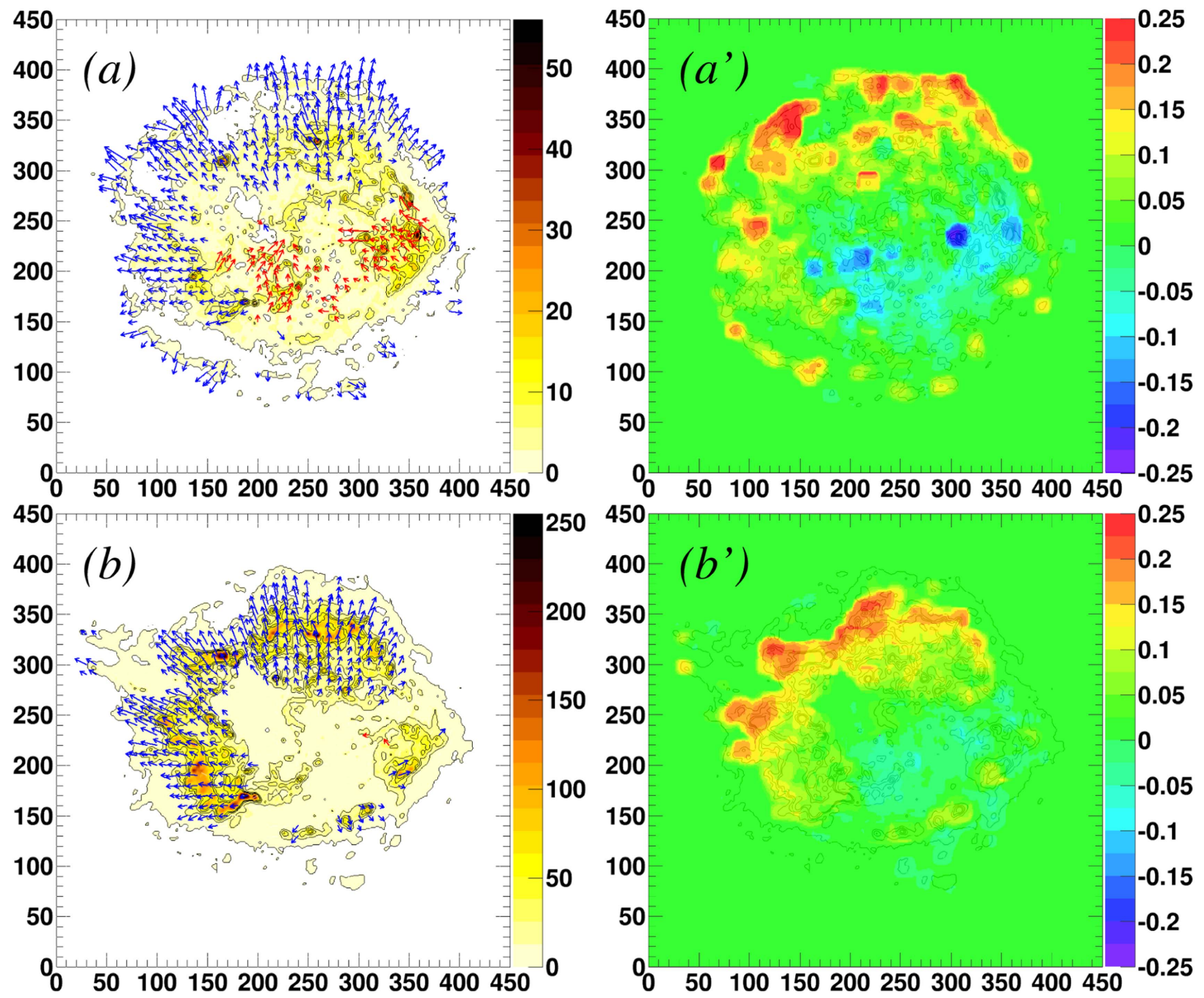

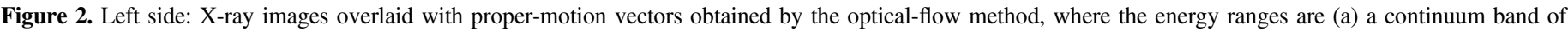

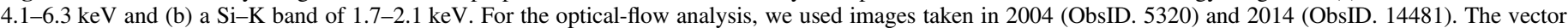

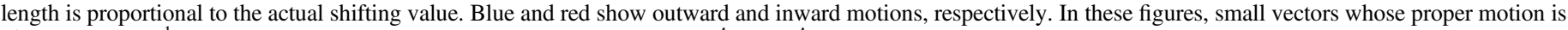

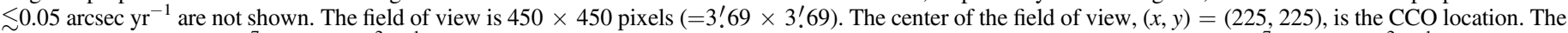

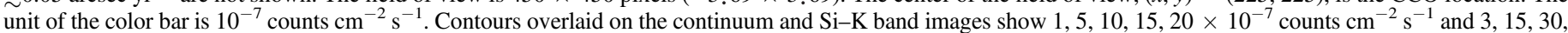

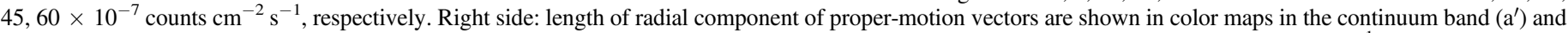

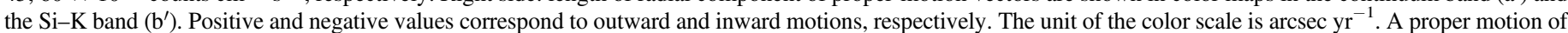
$0.15 \operatorname{arcsec}_{\mathrm{yr}^{-1}}$ corresponds to $\sim 2400 \mathrm{~km} \mathrm{~s}^{-1}$ at the distance of $3.4 \mathrm{kpc}$.

where $n_{i, j}$ is the counts in pixel $(i, j)$ of the image in each epoch, and $m_{i, j}$ is the model counts based on the 2004 image. The fitting errors can be estimated in the usual manner because the statistical distribution of $\Delta C=C-C_{\min }$ is similar to that of $\chi^{2}$ (Cash 1979).

The fitting results are summarized in Figure 3 and Table 3. We found that the best-fit positions of those filaments are gradually shifting from the outside to the inside with large proper-motion values: 0 ". $129-0$ ". $219 \mathrm{yr}^{-1}$ for the $\mathrm{W}$ filaments and $0.213-0.235 \mathrm{yr}^{-1}$ for the $\mathrm{C}$ filaments. The best-fit $x, \mathrm{y}$ shifts and errors listed in Table 3 were determined by chisquare fitting with a linear function, as shown in Figure 3. In the fitting, the slope provides the $x, y$ shift (arcsec per year) with the error coming from $\Delta \chi^{2}=1.0$. Using the values, we determined the proper motions and errors (see the Appendix for the method). For the assumed distance of $3.4 \mathrm{kpc}$ (Reed et al. 1995) to the remnant, we estimate the filament velocities to be $\sim 2100-3800 \mathrm{~km} \mathrm{~s}^{-1}$.
In the radio band, the kinematics of 304 knot structures over the entire remnant had been reported previously (Anderson \& Rudnick 1995). This work showed that the motions of many western knots deviated from that of knots in other regions and that some western knots were actually moving inward (see Figures 5 and 6 in Anderson \& Rudnick 1995). In the X-ray band, continuum-dominated filaments with an inward motion were also found at azimuths between $170^{\circ}$ and $300^{\circ}$ (DeLaney et al. 2004). These features correspond to the inward shocks we found.

\subsection{Flux Variations}

Here we investigate the X-ray light curves of the inwardmoving filaments. First we extracted spectra from each epoch for each inward-shock filament using the CIAO specextract command. For the source regions, we used ellipse 
Table 3

Proper Motions and Spectral Parameters of the Inward-shock Filaments in Cassiopeia A

\begin{tabular}{|c|c|c|c|c|c|c|c|}
\hline \multirow[b]{2}{*}{ id } & \multicolumn{5}{|c|}{ Imaging Analysis } & \multicolumn{2}{|c|}{ Spectral Parameters in 2004} \\
\hline & $\begin{array}{l}\text { Center of the Model Frame } \\
\text { R.A., Decl. (epoch 2004) }\end{array}$ & $\begin{array}{l}\text { Mean Shift: } \Delta x, \Delta y \\
\quad\left(\operatorname{arcsec} \mathrm{yr}^{-1}\right)\end{array}$ & $\begin{array}{l}\text { Proper Motion } \\
\left(\operatorname{arcsec}^{\mathrm{b}} \mathrm{yr}^{-1}\right)\end{array}$ & $\begin{array}{l}\text { velocity } \\
\left(\mathrm{km} \mathrm{s}^{-1}\right)\end{array}$ & $\begin{array}{c}\text { Angle } \\
\text { (degree) }\end{array}$ & Photon Index & $\begin{array}{r}\text { Flux in } 4.2-6 \mathrm{keV} \\
\left(10^{-13} \mathrm{erg} \mathrm{cm}^{-2} \mathrm{~s}^{-1}\right)\end{array}$ \\
\hline W1 & $23^{\mathrm{h}} 23^{\mathrm{m}} 12^{\mathrm{s}} \cdot 070,+58^{\circ} 49^{\prime} 23^{\prime \prime} 64$ & $-0.111 \pm 0.026,+0.077 \pm 0.027$ & $0.132 \pm 0.027$ & $2130 \pm 440$ & $145 \pm 11$ & $2.11_{-0.08}^{+0.05}$ & $5.70 \pm 0.04$ \\
\hline W2 & $23^{\mathrm{h}} 23^{\mathrm{m}} 11^{\mathrm{s}} .126,+58^{\circ} 48^{\prime} 56^{\prime \prime} 07$ & $-0.180 \pm 0.026,-0.089 \pm 0.027$ & $0.200 \pm 0.026$ & $3210 \pm 420$ & $206 \pm 8$ & $2.00_{-0.05}^{+0.03}$ & $19.79 \pm 0.07$ \\
\hline W3 & $23^{\mathrm{h}} 23^{\mathrm{m}} 17^{\mathrm{s}} \cdot 525,+58^{\circ} 48^{\prime} 50^{\prime \prime} 73$ & $-0.190 \pm 0.027,-0.113 \pm 0.027$ & $0.219 \pm 0.027$ & $3540 \pm 440$ & $211 \pm 7$ & $2.18_{-0.08}^{+0.05}$ & $6.65_{-0.03}^{+0.05}$ \\
\hline $\mathrm{C} 1$ & $23^{\mathrm{h}} 23^{\mathrm{m}} 31^{\mathrm{s}} \cdot 829,+58^{\circ} 48^{\prime} 29^{\prime \prime} 70$ & $+0.215 \pm 0.026,-0.013 \pm 0.027$ & $0.213 \pm 0.027$ & $3440 \pm 440$ & $356 \pm 7$ & $2.64_{-0.09}^{+0.06}$ & $6.56_{-0.05}^{+0.08}$ \\
\hline $\mathrm{C} 2$ & $23^{\mathrm{h}} 23^{\mathrm{m}} 29^{\mathrm{s}} \cdot 676,+58^{\circ} 48^{\prime} 30^{\prime \prime} \cdot 20$ & $+0.214 \pm 0.027,+0.101 \pm 0.027$ & $0.235 \pm 0.027$ & $3800 \pm 440$ & $25 \pm 6$ & $2.24_{-0.07}^{+0.05}$ & $8.41_{-0.07}^{+0.05}$ \\
\hline
\end{tabular}

Notes.

${ }^{\text {a }}$ All of the errors in this table show a $68 \%$ confidence level $\left(\Delta \chi^{2}=1.0\right)$. For the image analyses, the systematic uncertainties are also included.

$\mathrm{b}$ The proper motions and the errors are estimated using the Rice distribution (see the Appendix).

${ }^{\mathrm{c}}$ The distance to the remnant was assumed to be $3.4 \mathrm{kpc}$.

regions that included the bright filament structures. These regions were shifted with time, taking the proper-motion effects into account. The background regions were selected from nearby low-brightness regions. The background-subtracted spectra were fitted in the $4.2-6 \mathrm{keV}$ band using a powerlaw model in Xspec 12.9.0n (Arnaud 1996).

We summarize the fitting results in Figure 4. For all of the western filaments, over the $\sim 14$ yr span of observed X-ray fluxes increased by up to $\sim 40 \%-50 \%$ and afterward decreased to the initial flux level from epoch 2000. Background photons in the extracted spectrum are less than $30 \%$ of the total and fluctuate by $\lesssim \pm 20 \%$ (see the black dashed lines in Figure 4). The change in the background rate from a uniform level could introduce a fluctuation in the filament's X-ray flux of at most $\sim 9 \%$. Even given this effect, significant time variations of the $\mathrm{X}$-ray flux are still present. Time intervals of the monitoring observations would mainly influence on the estimation accuracy of variable timescales. The intervals are $\sim 2 \mathrm{yr}$ from 2000 to 2007 and $\sim 1$ yr from 2007 to 2014. Therefore, the uncertainty of estimated timescales would be $\pm 2 \mathrm{yr}$ for the W1 and W2 filaments and \pm 1 yr for the W3 and W4 filaments. Even taking these errors into consideration, the timescales are very short. For regions W1 and W2, the timescales of the flux increase up to the maximum level $\left(t_{\text {in }}\right)$ and decrease down to the initial level $\left(t_{\mathrm{dec}}\right)$ are $t_{\mathrm{in}} \sim t_{\mathrm{dec}}=4 \pm 2 \mathrm{yr}$. For regions W3 and $\mathrm{W} 4$, the increase times are longer than those at the $\mathrm{W} 1$ and W2 filaments. These filaments have a similar light curve, although the W4 filament's shows a slightly complex shape. As a representative of these, the $\mathrm{W} 3$ filament shows $t_{\text {in }}=9 \pm 1 \mathrm{yr}$ and $t_{\mathrm{dec}}=4 \pm 1 \mathrm{yr}$. On the other hand, regions $\mathrm{C} 1$ and $\mathrm{C} 2$ show little to no sudden change of flux; rather, their fluxes seem to be gradually decreasing by $\sim 20 \%-30 \%$ over a $\sim 14 \mathrm{yr}$ time span. The variability of the nonthermal X-ray flux is strongly related to the magnetic field (e.g., Uchiyama et al. 2007). The differences of the variable timescales seem be related to differences in the magnetic field strength at each region (this is discussed in Section 4.2).

Patnaude \& Fesen (2007) and Uchiyama \& Aharonian (2008) have already shown X-ray variations in small structures of the remnant using the first three epochs of the Chandra observations. Regions W1 and W2 are nearly the same as regions R4 in Patnaude \& Fesen (2007) and H in Uchiyama \& Aharonian (2008), respectively. Therefore, these are follow-up results for the past observations, and the time variations are almost consistent with each other in the same time interval.

Spectral parameters in 2004 are summarized in Table 3. We were not able to detect a significant time variation in the photon indices.

\subsection{Thickness of the Inward-shock Filaments}

We evaluate the spatial extent of the inward-shock filaments using Chandra's high angular resolution of $\sim 0$ ". 5 (FWHM). We found all filament widths to be in the range $2^{\prime \prime}-4^{\prime \prime}$ (Figure 5), which is very similar to the widths of the forwardshock filaments (1".5-4"; Vink \& Laming 2003). The angular resolution of the Wolter type-I optics used for the Chandra's mirrors degrades at off-axis field angles. However, at the offaxis location of the inward-shock filaments $\left(2^{\prime}-3^{\prime}\right)$, the angular resolution is nearly consistent with that at the on-axis position. ${ }^{13}$ Therefore, we are justified in ignoring the additional blur introduced by the off-axis effects for the shock thickness evaluation. At the remnant's distance of $3.4 \mathrm{kpc}$, the observational thickness is estimated to be $\sim 0.03-0.07 \mathrm{pc}$. We can estimate a synchrotron loss time from the widths by combining them with the plasma flow speed away from the shock front (see Section 4.2 for this discussion).

\section{Discussion}

We have shown that some bright filaments are moving inward with velocities of $\sim 2100-3800 \mathrm{~km} \mathrm{~s}^{-1}$ using all the monitoring data taken by Chandra from 2000-2014. These filaments cannot be located at the forward-shock position even when projection is considered because the forward shock must move outward. In addition, we found that those filaments are cospatial with hard X-ray hot spots seen by NuSTAR, and additionally that these filaments show flux variations (both increasing and decreasing) on timescales of a few years. These results imply that the hard X-ray emission is related to

\footnotetext{
13 See http://cxc.harvard.edu/proposer/POG/html/chap4.html for the angular resolutions at off-axis angles.
} 

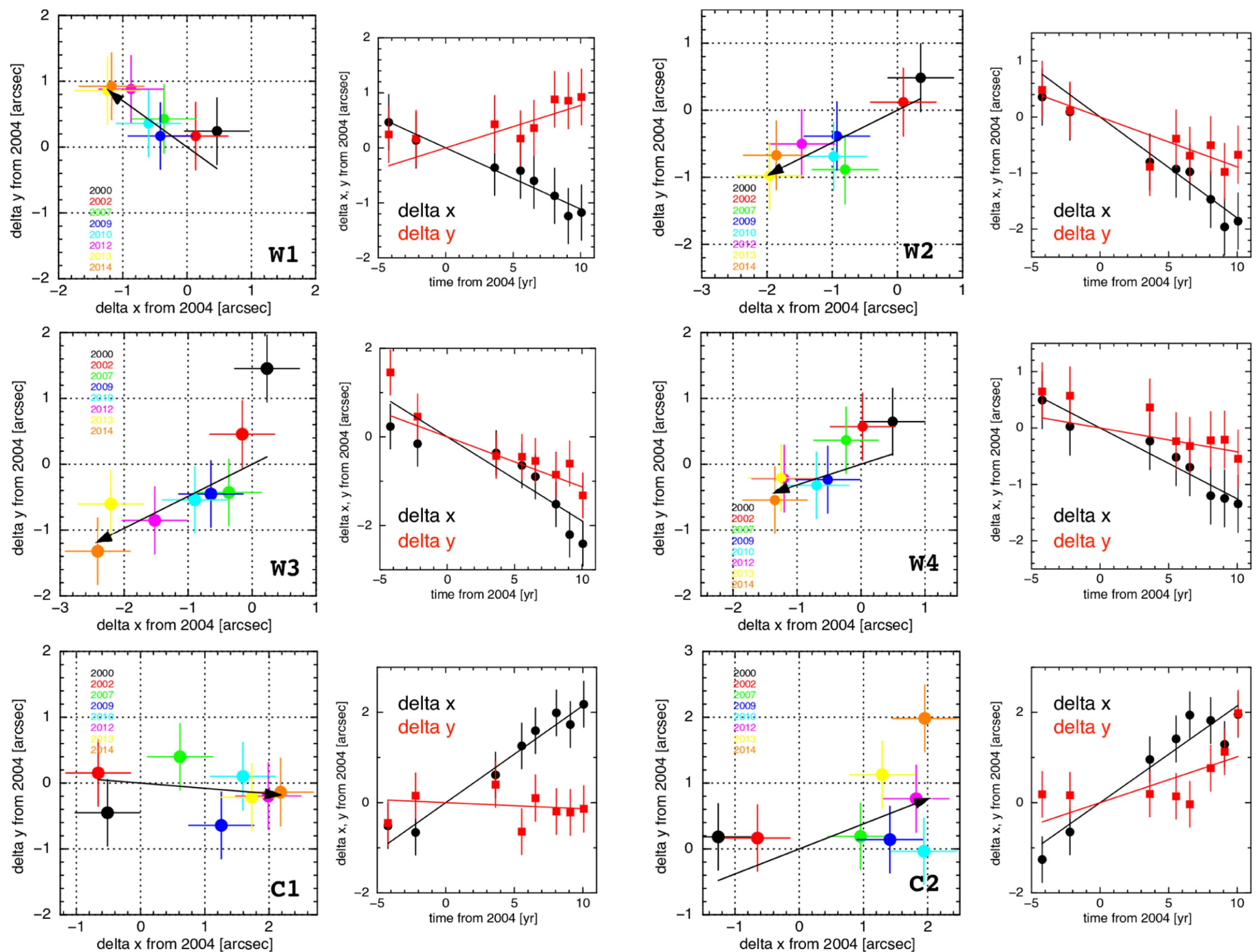

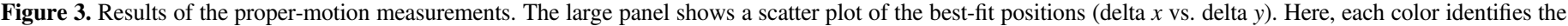

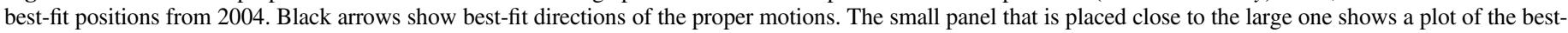

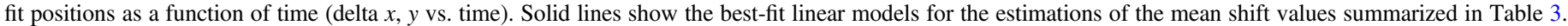

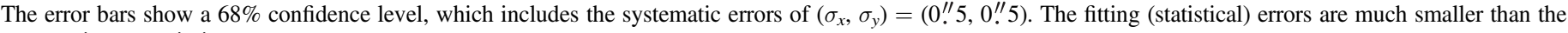
systematic uncertainties.

nonthermal emission from accelerated electrons at the inwardshock regions where the magnetic field is high amplified. These results hold the hope of advancing our understanding of the mysterious nonthermal X-ray emission at the interior of Cassiopeia $\mathrm{A}$ and understanding the particle acceleration that is taking place at these shocks. In this section, we discuss the implications of our results on the physical conditions at the inward shocks (Section 4.1), the process of particle acceleration there (Section 4.2), and the origin of the inward shocks themselves (Section 4.3).

\subsection{Inward Shock Conditions}

Here we make the plausible assumption based on the location of the inward shocks (see Figure 1) that they are propagating through the inner ejecta. Thus their actual shock velocities in the rest frame of the ejecta should be higher than what we measure from the proper motions. Here, we define the shock velocity $v_{s}$ as

$$
v_{s} \equiv v_{\mathrm{ej}}-v=\frac{R_{\mathrm{ej}}}{t}-v,
$$

where $v_{\mathrm{ej}}, v, \operatorname{Rej}$, and $t$ are the ejecta velocity, the velocity we measure, the typical ejecta radius, and the age of the remnant, respectively.

First, we consider the case where the inward shocks are propagating into cool, freely expanding ejecta. We adopt a value of $R_{\mathrm{ej}} \sim 5 \times 10^{18} \mathrm{~cm}$, which was estimated from the typical reverse-shock radius at the distance of $3.4 \mathrm{kpc}$ (Gotthelf et al. 2001) and then obtained an ejecta speed of $v_{\mathrm{ej}} \sim$ $4,900 \mathrm{~km} \mathrm{~s}^{-1}$ for $t \sim 320$ years (the remnant's age in 2000). The inward shocks have measured proper-motion velocities $v$ from $-2100 \mathrm{~km} \mathrm{~s}^{-1}$ to $-3,800 \mathrm{~km} \mathrm{~s}^{-1}$, leading to shock velocity estimates of $v_{s} \sim 7000-8700 \mathrm{~km} \mathrm{~s}^{-1}$. Next, we consider a case where the inward shocks are propagating into previously shocked, and therefore hot, ejecta. In this case, $v_{\mathrm{ej}}$ corresponds to the bulk expansion velocity of the ejecta. 

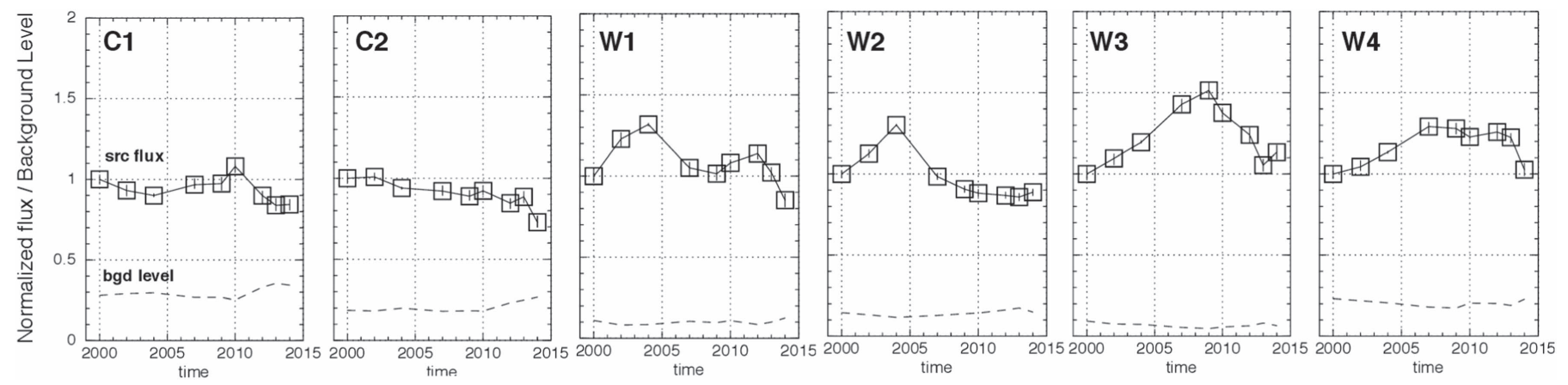

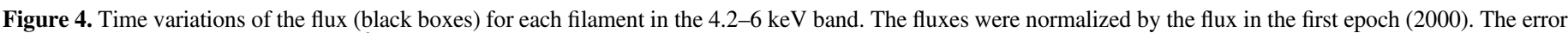
bars show $68 \%$ confidence level $\left(\Delta \chi^{2}=1.0\right)$. Broken lines show background levels in each epoch.

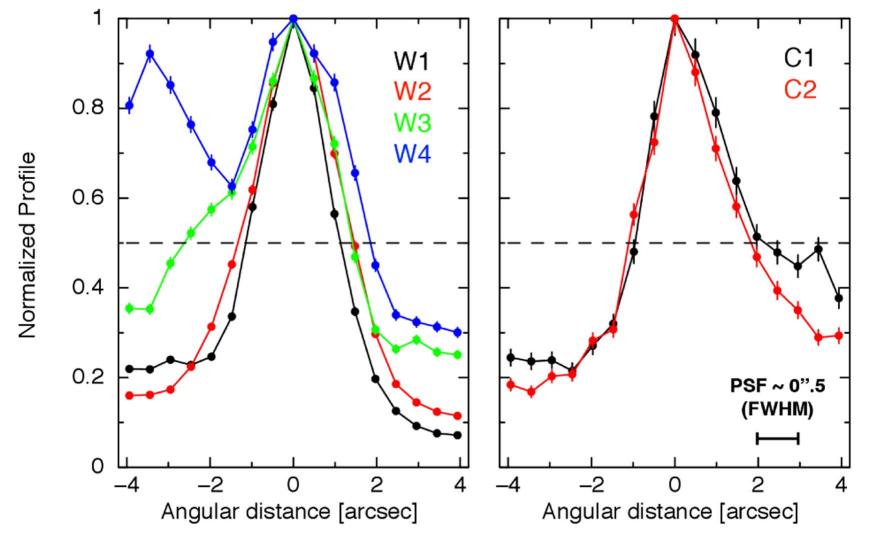

Figure 5. Emission profiles of the inward-shock filaments in the west (left) and center (right). The profiles were extracted from 15 pixels wide using a 2004 image in the $4.2-6 \mathrm{keV}$ band and were normalized by the peak intensities. The positive direction in the angular distance shows a direction to the outside of the remnant. The scale bar in the right panel shows the Chandra's angular resolution, $\sim 0$." 5 (FWHM).

DeLaney et al. (2004) estimated an expansion rate of $0.2 \% \mathrm{yr}^{-1}$ for the ejecta component. Using this value and the same value of $R_{\mathrm{ej}}$ as above, the expansion velocity is estimated to be $\sim 3,000 \mathrm{~km} \mathrm{~s}^{-1}$ (see also Holt et al. 1994; Willingale et al. 2002; Morse et al. 2004; DeLaney et al. 2010 for the ejecta velocity). Now the shock velocities we infer are $v_{s} \sim 5100-6800 \mathrm{~km} \mathrm{~s}^{-1}$. In either case, the intrinsic shock velocity of the inward shocks is some $\sim 1-2$ times higher than that of the forward shocks (4200-5200 $\mathrm{km} \mathrm{s}^{-1}$; Patnaude \& Fesen 2009).

The temperature of the medium (cool or hot plasma) into which the shock wave is propagating changes the conditions of the shock. Assuming first the cool ejecta as the medium, the sound speed (a few tens $\mathrm{km} \mathrm{s}^{-1}$ ) is much lower than the shock velocity $\left(\sim 7000-8700 \mathrm{~km} \mathrm{~s}^{-1}\right)$, implying a high Mach number, $\mathcal{M} \gtrsim 100$. In this case, the shock compression ratio is $r=4$. On the other hand, the Mach number of the shock could be much lower if the inward shock is propagating into hot plasma with a high sound speed. Using a typical value for the electron temperature for Cassiopeia A ( $2 \mathrm{keV}$, e.g., Hwang \& Laming 2012), the sound speed $c_{s}$ and Mach number $\mathcal{M}$ are estimated to be $c_{s} \sim 730 \mathrm{~km} \mathrm{~s}^{-1}$ and $\mathcal{M} \sim 7-8$ (for $v_{s} \sim 5100-6800 \mathrm{~km} \mathrm{~s}^{-1}$ ). Then the compression ratio $r=\frac{(\gamma+1) \mathcal{M}^{2}}{(\gamma-1) \mathcal{M}^{2}+2}$ is estimated to be $\sim 3.8$ for $\gamma=5 / 3$ and $\mathcal{M}=7-8$, which is close to the high Mach number limit of $r=4$. In contrast, the forward shock propagates though the interstellar medium (ISM), where both a high Mach number and a high compression ratio are generally expected. We here note that effects of particle acceleration can produce a shock with a compression ratio greater $r=4$ (e.g., Berezhko \& Ellison 1999). As relativistic particles are produced and significantly contribute to the total pressure, the shocked plasma becomes more compressible $(\gamma \rightarrow 4 / 3, r \rightarrow 7)$. Additionally, the escape of the highest energy particles would also further compress the shock by carrying off energy, analogous to that case of radiative shocks (e.g., Eichler 1984; Berezhko \& Ellison 1999; Ellison et al. 2005).

A further complication for the shocked plasma in the remnant concerns the possibility that the electron and ion temperatures are not equilibrated. In the extreme case, the ions possess virtually all of the thermal energy. Laming (2001) has calculated the time variation of the ion and electron temperatures for reverse shocks into pure oxygen ejecta for Cassiopeia A. Based on his results, we assume a plasma temperature of $\sim 46 \mathrm{keV}$, which is the ion temperature in the shocked plasma $150 \mathrm{yr}$ after explosion. ${ }^{14}$ Here the sound speed $c_{s}$ and the Mach number $\mathcal{M}$ are estimated to be $c_{s} \sim 3500 \mathrm{~km} \mathrm{~s}^{-1}$ and $\mathcal{M} \sim 1.5-1.9$. Also, the compression ratio is estimated to be $\sim 2$ for $\gamma=5 / 3$ and $\mathcal{M}=1.7$. However, it is possible that particles would not accelerated in this case because the Mach number is below the critical value of $\mathcal{M}<\sqrt{5}$ for particle acceleration (Vink \& Yamazaki 2014). Columns 1 and 2 of Table 4 summarize the compression ratios and the shock velocities for the several cases we study here. Given the current state of knowledge, all of these cases offer plausible scenarios for the forward and inward shocks.

Determining the shock conditions more accurately requires measuring the ion temperatures via, e.g., thermal line broadening in the X-ray spectra. From the Chandra grating spectroscopy of some bright knots (e.g., Lazendic et al. 2006), ion temperatures were estimated to be quite low (the observed lines were narrow). Therefore, the high Mach number scenario may be realistic for at least some dense features. On the other hand, for diffuse extended sources like Cassiopeia A, it is difficult to measure the line broadening accurately using grating spectroscopy. In the future, observations with a X-ray calorimeter would be more powerful. For example, the high energy resolution $(\mathrm{FWHM} \simeq$ $5 \mathrm{eV}$ ) calorimeter on board the Hitomi satellite measured line broadening of $\sim 200 \mathrm{~km} \mathrm{~s}^{-1}$ in the Perseus cluster (Hitomi Collaboration et al. 2016, 2017) and would have revealed much about the thermal broadening in SNRs, too. We await the launch of X-ray Astronomy Recovery Mission (XARM), the recovery

\footnotetext{
${ }^{14}$ See Table 3 and Figure 3 in Laming (2001) for more details. The plasma parameters in this case explained well what the typical values in the remnant are (e.g., $k_{\mathrm{e}} T \sim 2.3 \mathrm{keV}, n_{\mathrm{e}} t \sim 7 \times 10^{10} \mathrm{~cm}^{3} \mathrm{~s}^{-1}$ ).
} 
Table 4

Summary of Diffusion and Acceleration Parameters Estimated for the Forward Shock and the Reverse Shock

\begin{tabular}{|c|c|c|c|c|c|c|c|}
\hline Compression Ratio & Shock Velocity & $E_{\gamma, \mathrm{cut}, \mathrm{keV}}$ & $B$ & $k_{0}$ & $E_{\mathrm{e}, \max }$ & $\tau_{\text {syn }}$ & $\tau_{\mathrm{acc}}$ \\
\hline \multicolumn{8}{|l|}{ Forward Shock } \\
\hline$r=4$ & $4200-5200 \mathrm{~km} \mathrm{~s}^{-1(2)}$ & $2.3 \mathrm{keV}^{(1)}$ & $0.1 \mathrm{mG}(2),(3)$ & $1.1-1.6$ & $34 \mathrm{TeV}$ & 37 years & 63 years \\
\hline " & " & " & $0.5 \mathrm{mG}(4)$ & " & $15 \mathrm{TeV}$ & 3 years & 6 years \\
\hline$r=7$ & "1 & " & $0.1 \mathrm{mG}(2),(3)$ & $0.7-1.1$ & $34 \mathrm{TeV}$ & 37 years & 63 years \\
\hline " & " & " & $0.5 \mathrm{mG}(4)$ & " & $15 \mathrm{TeV}$ & 3 years & 6 years \\
\hline \multicolumn{8}{|l|}{ Inward Shock } \\
\hline$r=4$ (into cool ejecta) & $7000-8700 \mathrm{~km} \mathrm{~s}^{-1 \text { (this work) }}$ & $1.3 \mathrm{keV}^{(1)}$ & $0.5 \mathrm{mG}^{(5),(\text { this work })}$ & $5.3-8.2$ & $11 \mathrm{TeV}$ & 4 years & 7 years \\
\hline " & " & " & $1.0 \mathrm{mG}^{(5),(\text { this work })}$ & " & $8 \mathrm{TeV}$ & 2 years & 3 years \\
\hline$r=3.8$ (into $2 \mathrm{keV}$ plasma) & $5100-6800 \mathrm{~km} \mathrm{~s}^{-1 \text { (this work) }}$ & " & $0.5 \mathrm{mG}^{(5),(\text { this work })}$ & $2.9-5.1$ & $11 \mathrm{TeV}$ & 4 years & 7 years \\
\hline " & $\prime \prime$ & " & $1.0 \mathrm{mG}^{(5),(\text { this work })}$ & " & $8 \mathrm{TeV}$ & 2 years & 3 years \\
\hline$r=2$ (into $46 \mathrm{keV}$ plasma) & " & " & $0.5 \mathrm{mG}^{(5),(\text { this work) }}$ & $3.7-6.6$ & $11 \mathrm{TeV}$ & 4 years & 7 years \\
\hline " & " & " & $1.0 \mathrm{mG}^{(5),(\text { this work })}$ & " & $8 \mathrm{TeV}$ & 2 years & 3 years \\
\hline
\end{tabular}

Note.

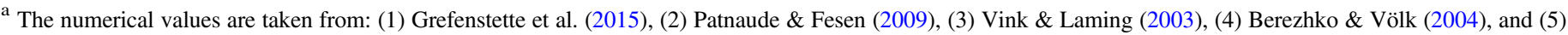
Uchiyama \& Aharonian (2008).

mission for the Hitomi satellite, to reveal the shock condition more accurately in Cassiopeia A.

\subsection{The Diffusion Coefficient and Particle Acceleration in Cassiopeia A}

In DSA, particles are accelerated by scattering multiple times across the shock front. Particle diffusion is characterized by the level of magnetic field turbulence. If the strength of the turbulent field is close to the unperturbed magnetic field strength (Bohm regime: $\delta B \sim B$ ), then particles can be efficiently scattered and accelerated (see a review in Reynolds 2008). This situation is often assumed for particle acceleration in SNRs, although it might not be so in some cases (e.g., Parizot et al. 2006; Eriksen et al. 2011). In the following, we follow Parizot et al. (2006), who characterize departures

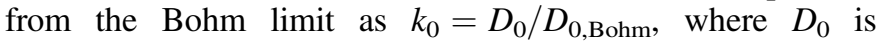
the diffusion coefficient at the electron cut-off energy. This parameter can be expressed in terms of the photon cut-off energy $\left(E_{\gamma, \text { cut }}=h \nu_{\text {cut }}\right)$, shock velocity $\left(V_{3}\right.$, in units of $\left.1000 \mathrm{~km} \mathrm{~s}^{-1}\right)$, and the compression ratio $r$ as

$$
k_{0}=0.14 E_{\gamma, \mathrm{cut}, \mathrm{keV}}^{-1} V_{3, \text { shock }}^{2} \frac{16(r-1)}{3 r^{2}} .
$$

The NUSTAR spectra above $15 \mathrm{keV}$ showed photon cut-off energies for the forward shock and the reverse shock of $2.3 \mathrm{keV}$ and $1.3 \mathrm{keV}$, respectively (Grefenstette et al. 2015). We here assume the shock velocity and the compression ratio at the forward shock and the inward shock listed in Table 4, which are estimated in the Section 4.1. For the forward shock, the diffusion parameter was $k_{0} \lesssim 1.6$, which is close to the Bohm regime, in both of the compression ratios $(r=4,7)$. On the other hand, the inward shock shows departures from Bohm diffusion: $k_{0} \gtrsim 3$ (Table 4). Stage et al. (2006) have already suggested a similar $k_{0}$ difference between the forward shock and the inward shock in their map of the electron cut-off frequencies in Cassiopeia A. Those authors estimated upper limits to the diffusion coefficient and found that the forward shock in the north, northeast, and southeast was close to the Bohm limit. These results agree with ours.

Using the coefficient $k_{0}$ and other observational values, we can also estimate the electron maximum energy $E_{\mathrm{e}, \max }$, the synchrotron cooling timescale $\tau_{\text {syn }}$, and the acceleration timescale $\tau_{\text {acc }}$, as shown below (Parizot et al. 2006):

$$
\begin{gathered}
E_{\mathrm{e}, \max } \simeq(8.3 \mathrm{TeV}) \frac{4}{\sqrt{3}} \frac{\sqrt{r-1}}{r} k_{0}^{-1 / 2} B_{0.1}^{-1 / 2} V_{\mathrm{sh}, 3}, \\
\tau_{\mathrm{syn}} \simeq\left(1.25 \times 10^{3} \text { years }\right) E_{\mathrm{TeV}}^{-1} B_{0.1}^{-2}, \\
\tau_{\mathrm{acc}} \simeq(30.6 \text { years }) \frac{3 r^{2}}{16(r-1)} k_{0} E_{\mathrm{TeV}} B_{0.1}^{-1} V_{\mathrm{sh}, 3}^{-2},
\end{gathered}
$$

where $B_{0.1}$ and $E_{\mathrm{TeV}}$ are the magnetic field in units of $0.1 \mathrm{mG}$ and the electron energy in units of $10^{12} \mathrm{eV}$, respectively. At the forward-shock region, $B=0.08-0.16$ or $0.5 \mathrm{mG}$ have been estimated using the width of the rim of $1 .{ }^{\prime \prime} 5-4^{\prime \prime}$, and also the average magnetic field in the whole SNR was estimated to be $B>0.5 \mathrm{mG}$ (Vink \& Laming 2003; Berezhko \& Völk 2004). For $B \gtrsim 0.5 \mathrm{mG}$, the synchrotron cooling timescale becomes comparable to the variability timescale of $\lesssim 4$ yr at the reverseshock region (Uchiyama \& Aharonian 2008). Our results show almost the same timescale as that in Uchiyama \& Aharonian (2008). From these observational results, we assumed the typical magnetic fields at the forward shock and the reverse shock are $0.1-0.5 \mathrm{mG}$ and $0.5-1 \mathrm{mG}$, respectively. Using these parameters, we newly estimate the acceleration parameters for both the forward and inward shocks of Cassiopeia A (Table 4).

We find the maximum electron energy for the inward shock, $E_{\mathrm{e}, \max }=8-11 \mathrm{TeV}$ to be smaller than that of the forward shock $\left(E_{\mathrm{e}, \max }=15-34 \mathrm{TeV}\right)$, even though the shock velocity at the inward shock is higher. This is because the inward shock has larger $k_{0}$ and $B$ values. The variability timescales are also estimated to be shorter at the inward shock: $\tau_{\text {syn }} \sim 2-4 \mathrm{yr}$, $\tau_{\text {acc }} \sim 3-7$ years ( $e$-folding time), which is mainly due to the strong magnetic field of 0.5-1 mG. In Section 3.3, we found the flux variations with timescales (changing by $~ 40 \%-50 \%$ ) of $t_{\mathrm{dec}}=4 \pm 1 \mathrm{yr}$ and $t_{\mathrm{in}}=9 \pm 1 \mathrm{yr}$ for the W3 (and maybe also W4) region. These timescales are very similar to the predicted timescales for $B=0.5 \mathrm{mG}$. For regions $\mathrm{W} 1$ and $\mathrm{W} 2$, the timescales were estimated to be $t_{\text {in }} \sim t_{\text {dec }}=4 \pm 2 \mathrm{yr}$, which is also close to the timescale for a strong magnetic field of $0.5-1 \mathrm{mG}$. For regions $\mathrm{C} 1$ and $\mathrm{C} 2$, we found gradual flux declines of $\sim 20 \%-40 \%$ over $\sim 14$ yr. Here, $B \sim 0.2 \mathrm{mG}$ can explain well the flux-decline timescale. From these points, we 
note that differences of the variability timescales can be explained by difference in the magnetic field strength. In addition, we note that the time variation of spatially adjoining regions are similar to each other (see both Figures 1 and 3). This tendency may also imply something about the difference of the magnetic field from region to region. For example, the radial positions of the inward shocks, $R_{\mathrm{W} 1, \mathrm{~W} 2}>R_{\mathrm{W} 3, \mathrm{~W} 4}>R_{\mathrm{C} 1, \mathrm{C} 2}$, seem to be inversely related to the variability timescales, $t_{\mathrm{W} 1, \mathrm{~W} 2}<t_{\mathrm{W} 3, \mathrm{~W} 4}<t_{\mathrm{C} 1, \mathrm{C} 2}$. This tendency might suggest that magnetic field amplification has a dependence on the radial location of the shock within the remnant. In contrast, the variability timescales at the forward shock are much longer than at the inward shock. Sato et al. (2017) have shown the time variation of X-ray flux at the forward-shock region is not significant: $0.00 \% \pm 0.07 \% \mathrm{yr}^{-1}$. The constant flux can be explained by the balance between acceleration and cooling even for both cases of high and low magnetic fields in Table 4 (see also Katsuda et al. 2010). We leave investigations into specific scenarios to account for the rapid flux changes that we see in some of the inward shocks for future works.

The synchrotron cooling timescale can be also estimated from the inward-shock width, $\sim 0.03-0.07 \mathrm{pc}$, presented in Section 3.4. The accelerated electrons are advected away from the shock at a downstream velocity $V_{\mathrm{d}}=V_{\mathrm{sh}} / r$ during the synchrotron timescale, where $r$ is the shock compression ratio. The size of the advection region is thus given by $\Delta R_{\mathrm{adv}}=V_{\mathrm{d}} \times \tau_{\mathrm{syn}}$, where $\Delta R_{\mathrm{adv}}$ is the advection width. Although particle diffusion also expands the width, we simply constrain the observational width as $\Delta R_{\text {obs }} \geqslant V_{\mathrm{d}} \times \tau_{\text {syn }} \times P$, where $P$ is a projection factor. In the ideal case of a spherical shock, the projection factor is $P=4$.6. Parizot et al. (2006) described the relation between the synchrotron cooling timescale and the observational width as

$$
\tau_{\text {syn }} \leqslant \frac{\Delta R_{\text {obs }}}{V_{\mathrm{d}}} \simeq(8.5 \text { years }) \times \frac{r}{4} \times \bar{P}^{-1} \times V_{\mathrm{sh}, 3}^{-1} \times \Delta R_{\mathrm{obs},-2},
$$

where $\bar{P}$ and $\Delta R_{\text {obs, }-2}$ are defined as $\bar{P}=P / 4.6$ and $\Delta R_{\mathrm{obs}} \equiv$ $\Delta R_{\mathrm{obs},-2} \times 10^{-2} \mathrm{pc}$, respectively. Using Equation (7) and the estimated parameters in Table 4, the relation between the cooling time and the shock width was estimated (Figure 6). For a typical inward-shock width of $0.05 \mathrm{pc}$, the cooling timescale in the inward shock is $\lesssim 3-5 \mathrm{yr}$, which is consistent with the observed decay timescale and the predicted cooling timescale in the case of $B=0.5-1 \mathrm{mG}$ (Table 4). For the forward shock, the timescale is longer $(\sim 10 \mathrm{yr})$ than that of the inward shock. This tendency is the same as the other observational timescale estimates (Table 4).

Particle acceleration at reverse shocks have been studied in some theoretical works (e.g., Ellison et al. 2005; Telezhinsky et al. 2012, 2013). For all of the models, the maximum energy reached by particles at the reverse shock is always lower than at the forward shock. Also, recent deep $\gamma$-ray observations of Cassiopeia A with Major Atmospheric Gamma-ray Imaging Cherenkov Telescope (MAGIC) have showed a clear exponential cutoff at $\sim 3.5 \mathrm{TeV}$ (Guberman et al. 2017), which implies only modest cosmic-ray acceleration to very high energy for the hadronic scenario. From the observations, Cassiopeia $\mathrm{A}$ is not considered to be a PeVatron (PeV accelerator) at its present age. Both theoretical and observational studies suggest that particle acceleration at the reverse shock (inward shock) in Cassiopeia A could not produce the

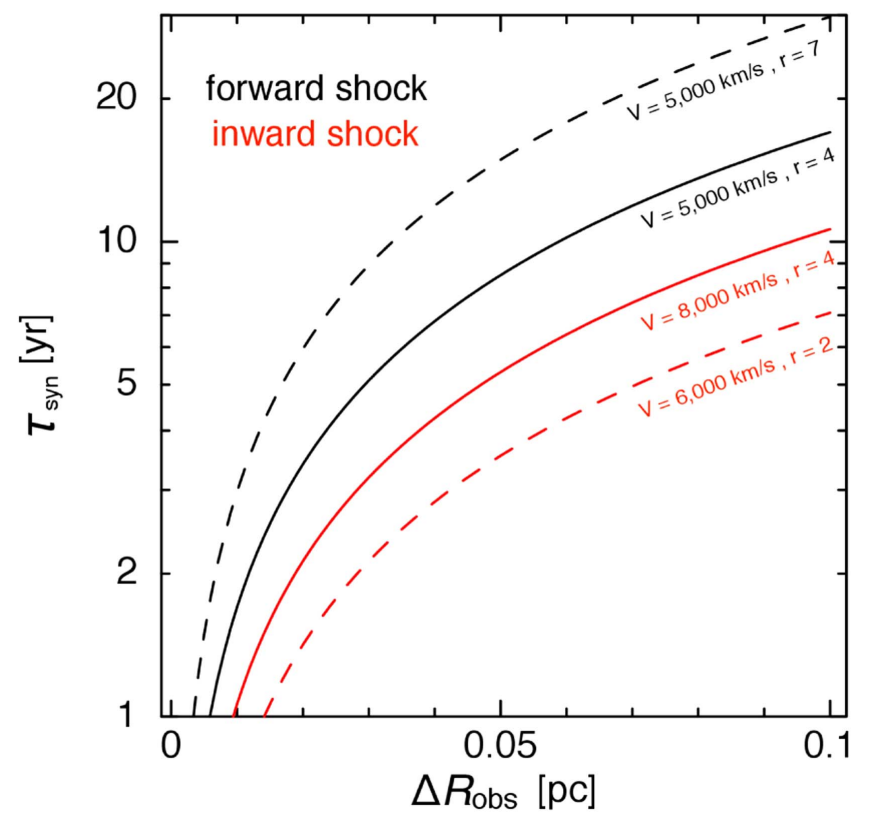

Figure 6. Synchrotron cooling timescale and observational shock width for the forward shock (black) and the inward shock (red) estimated from typical parameters in Table 4.

high energy particles (protons) up to "knee" energy $(\sim 3 \times$ $10^{15} \mathrm{eV}$ ), even if the shock has as high a shock velocity as that shown in Section 4.1. On the other hand, the reverse-shock region is propagating into a metal-rich environment produced by supernova nucleosynthesis. If such elements were accelerated by the reverse shocks, then the cosmic-ray composition would be affected. Therefore, particle acceleration at reverse shocks is important for understanding cosmic-ray abundances.

\subsection{The Origin of the Inward Shocks}

For the SNR expansion into an ambient medium with a constant or gradually declining density profile (e.g., ISM, circumstellar medium), it is not possible to explain an inwardmoving reverse shock (in the observer frame) for an evolutional phase that describes Cassiopeia A. In such a young evolutionary phase, the reverse shock must expand outward. As an alternative explanation for the inward shocks, we consider a "reflection shock" produced by the forward shock interacting with a density jump in the ambient medium.

The reflection shock conditions can be calculated using a simple fluid equation (Hester et al. 1994). We here assume a density of ambient medium before the encounter with the density jump as $\rho_{0}$. The density jump $\rho_{1}$ is defined as $\rho_{1} \equiv \alpha$ $\rho_{0}$. Then, the forward-shock density $\rho_{2}$ is described as $\rho_{2}=4$ $\rho_{0}$ from the Rankine-Hugoniot relation. After the encounter, a contact discontinuity is made by the interaction. Upward materials are compressed by $4 \rho_{1}$, and then downward materials are pushed back (see also Inoue et al. 2012). Figure 7 shows the estimate of the reflection shock speed using Hester et al. (1994). We assumed the forward shock is interacting with the density jump with the speed of $5,200 \mathrm{~km} \mathrm{~s}^{-1}$. In order to explain the inward-shock velocities, we require that the forward shock interacts with a surrounding material whose density is \5-8 times higher than that of a ambient medium elsewhere.

A local molecular cloud would be a good candidate for explaining such an enhanced density structure around the 


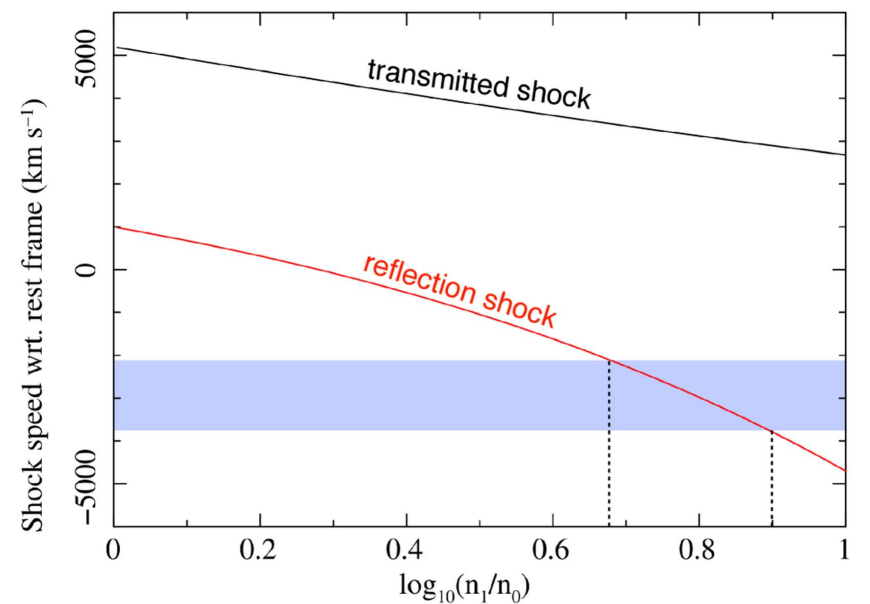

Figure 7. Estimate of the reflection shock velocity and the density of the interacting cloud in the case of Cassiopeia A, solving the fluid equation in Hester et al. (1994). Black and red curves show the velocities of the transmitted shock (that is propagating through the cloud) and reflection shock, respectively. The blue area shows the range of the velocities of the western filaments in this work: $\sim 2100-3800 \mathrm{~km} \mathrm{~s}^{-1}$.

remnant. Millimeter observations in ${ }^{12} \mathrm{CO}$ and ${ }^{13} \mathrm{CO} \mathrm{J}=2-1$ (230 and $220 \mathrm{GHz}$ ) with the Heinrich Hertz Submillimeter Telescope indicated the existence of the molecular cloud around Cassiopeia A (Kilpatrick et al. 2014). In particular, the inward-shock regions seems to overlay with the fastest gas $\left(-50\right.$ to $\left.-45 \mathrm{~km} \mathrm{~s}^{-1}\right)$ and the slowest gas (-39 to $\left.-34 \mathrm{~km} \mathrm{~s}^{-1}\right)$. We show a schematic diagram of the shock-cloud interaction in Figure 8. As shown in this figure, the inward shocks seems to be radially shifting against the distribution of the molecular clouds. Here, we can estimate the time when the forward shock first hits the cloud and pushes the reflection shock into the ejecta. Dividing the distance between the forward shock and the inward shocks $(\sim 0.7$ pc; see Figure 8$)$ by their velocity difference of $\sim 7000 \mathrm{~km} \mathrm{~s}^{-1}$, it can be estimated to be $<100 \mathrm{yr}$ ago. This is much shorter than the age of the remnant (which is $\sim 350$ yr old), providing us with further support that the inward shocks are reflection shocks by the western cloud. The western filaments and also the filaments at the central position (e.g., C1, C2) might be also related to the shockcloud interaction. For example, the high-speed $\mathrm{CO}$ gas $(-46$ to $-49 \mathrm{~km} \mathrm{~s}^{-1}$ ) is concentrated in filamentary structure to the south and southeast of the remnant. The locations are very close to the positions of the central inward shocks (see right side of Figure 3 in Kilpatrick et al. 2014).

However, it is difficult to confirm the shock-cloud interaction. Kilpatrick et al. (2014) have argued for a shockcloud interaction around the western and southern rim using the broadened CO lines (see Figure 4 in Kilpatrick et al. 2014). As a matter of fact, their locations are a little different from the inward-shock positions. Future observations with the Nobeyama $45 \mathrm{~m}$ Telescope will be helpful for studying the shock interaction with the molecular cloud. ${ }^{12} \mathrm{CO}$ observations with the telescope would have good spatial resolution $\left(\sim 17^{\prime \prime}\right)$ that will be able to reveal more small structures and the relation with the X-ray distributions (T. Inaba et al. 2017, private communication). For example, in the case of $\mathrm{RX}$ J1713.7-3946, nonthermal X-rays that are enhanced around $\mathrm{CO}$ and $\mathrm{H}_{\mathrm{I}}$ clumps have been found (e.g., Sano et al. 2010, 2013, 2015). They suggested that the amplified magnetic field around the $\mathrm{CO}$ and $\mathrm{H}_{\mathrm{I}}$ clumps enhances the synchrotron X-rays

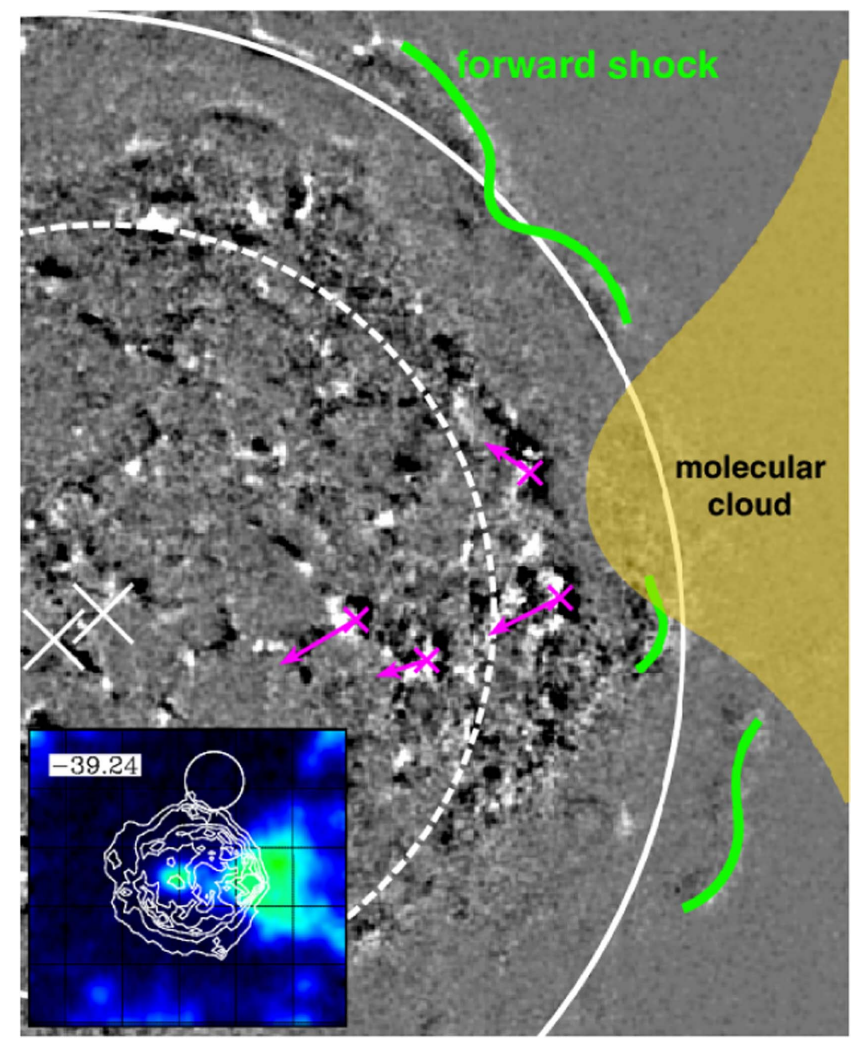

Figure 8. Schematic diagram of the shock-cloud interaction. The image show the same image as in Figure 1(a). Magenta arrows show the proper-motion directions of the inward shocks. The forward shocks are emphasized by thick green lines. Small and large circles indicate the radii of the reverse shock and forward shock (Gotthelf et al. 2001), respectively. Cross marks show the center positions of each circle. The center of the reverse-shock circle offsets to the northeast direction. The small screen set on left bottom shows the CO map with the velocity of $-39.24 \mathrm{~km} \mathrm{~s}^{-1}$ in Kilpatrick et al. (2014). A distance between the reverse shock and the forward shock is $\sim 0.7 \mathrm{pc}$ at the western region.

and possibly the acceleration of cosmic-ray electrons. In the theoretical view, the amplified magnetic field could be explained as one of features of the shock-cloud interaction. Inoue et al. (2012) have investigated cosmic-ray acceleration, assuming an interaction with clumpy interstellar clouds, using 3D magnetohydrodynamic simulations. They predicted a highly amplified magnetic field of $\sim 1 \mathrm{mG}$ caused by a turbulent shell due to the shock-cloud interactions. Then, the short-time X-ray variability was predicted at the same time. This supports our observational results in Cassiopeia A well, as discussed in the Section 4.2.

\section{Conclusion}

The bright, nonthermal X-ray emission in the interior of Cassiopeia $\mathrm{A}$ has been one of the most enigmatic features of the remnant since the earliest observations by Chandra. Even as basic a fact as the type of shock (e.g., forward shock, reverse shock, or something else) has remained obscure. In this paper, we put forth new evidence that the interior nonthermal emission originates in an "inward shock" through new analyses of archival Chandra and NUSTAR observations. We identified inward-moving filaments in the remnant's interior using monitoring data by Chandra from 2000 to 2014. The inwardshock positions are spatially coincident with the most intense hard (15-40 keV band) X-ray emission seen with NuSTAR. 
We measured the proper motions of the inward shocks, which equate to speeds of $\sim 2100-3800 \mathrm{~km} \mathrm{~s}^{-1}$ for a distance of $3.4 \mathrm{kpc}$ to the remnant. Assuming that the shocks are propagating through the expanding ejecta (which is itself moving outward), we determine that the shock velocities in the frame of the ejecta could reach up to $\sim 5100-8700 \mathrm{~km} \mathrm{~s}^{-1}$, which is $\sim 1-2$ times higher than that at the forward shock. Additionally, some of the inward-shock filaments showed flux variations (both increasing and decreasing) on timescales of just a few years. We find that the high shock velocity combined with a high magnetic field strength $(\sim 0.5-1 \mathrm{mG})$ in the reverseshock region can explain the nonthermal properties well. At the same time, we are able to constrain the diffusion coefficient and find that diffusion at the reverse shock is less efficient $\left(k_{0} \gtrsim 3\right)$ than that of the forward shock $\left(k_{0} \lesssim 1.6\right)$. Expressed in terms of magnetic field turbulence, we find (as do others) that the turbulence at the forward shock approaches the Bohm limit, while at the inward shocks turbulence is less well developed.

As to the nature of the inward shocks, we propose that they are "reflection shocks" caused by the forward shock's interaction with a density enhancement in the circumstellar medium. A density jump of a factor of $\gtrsim 5-8$ reproduces the observed inward-shock velocities. Previous works have shown evidence for a local molecular cloud on the western side of Cassiopeia A with some indications of an interaction between the cloud and the remnant's shock. Further investigations into the shock-cloud interaction will be useful to deepen our understanding of particle acceleration in Cassiopeia A.

We thank the Chandra and NuSTAR Operations, Software, and Calibration teams for support with the execution and analysis of these excellent observations. This work was supported by the Japan Society for the Promotion of Science (JSPS) KAKENHI Grant Nos. 16J03448 (T.S.), 16K17673 (S.K.), and 17K05395 (M.M.). Also, M.M. is supported by CREST. F.F. was supported, in part, by NASA under Grant NNX15AJ71G. This research was also supported in part by NASA grant NNX15AK71G to Rutgers University. J.P.H. acknowledges the hospitality of the Flatiron Institute, which is supported by the Simons Foundation. We thank Tetsuta Inaba, Hidetoshi Sano, Yasuo Fukui, Tsuyoshi Inoue, Ryo Iizuka, Takaaki Tanaka, Hiroyuki Uchida, Kuniaki Masai, Shiro Ikeda, Robert A. Fesen, and Daniel J. Patnaude for helpful discussions and suggestions in preparing this paper. We also thank the anonymous referee for comments that helped us to improve the manuscript. This work is partly supported by Leading Initiative for Excellent Young Researchers, MEXT, Japan.

Software: NuSTARDAS (v1.6.0), CIAO (version 4.6; Fruscione et al. 2006), OpenCV (v3.2.0; http://opencv.org), XSPEC (v12.9.0n; Arnaud 1996)).

\section{Appendix \\ Rice Distribution}

In our study, we determined the magnitude of proper-motion $\mu$ and its direction $\theta$ from measurements along the R.A. and decl. axes. To a sufficient accuracy, we can approximate our measurements as normally distributed in $X$ and $Y$ with means of $\mu \cos (\theta)$ and $\mu \sin (\theta)$ and variances of $\sigma^{2}$. This formulation assumes that the uncertainties are the same in both directions. Our naïve estimate for the proper motion, $p=\left(X^{2}+Y^{2}\right)^{1 / 2}$, follows the Rice distribution (see also Appendix I in Serkowski 1958), which has a probability density function
(PDF) given by

$$
f(p)=\frac{p}{\sigma^{2}} \exp \left(-\frac{p^{2}+\mu^{2}}{2 \sigma^{2}}\right) I_{0}\left(\frac{p \mu}{\sigma^{2}}\right),
$$

where $I_{0}$ is the modified Bessel function of the first kind with the order of zero. This distribution function is bounded at zero and is asymmetric, especially when $\mu / \sigma^{2}$ is small. Hence the naïve estimate for the magnitude of proper motion just introduced is biased high. In order to account for this bias, we use the Rice PDF.

The mean of the Rice PDF is

$$
\langle\mu\rangle=\sigma \sqrt{\pi / 2} \exp \left(-\mu^{2} / 2 \sigma^{2}\right)_{1} F_{1}\left(3 / 2 ; 1 ; \mu^{2} / 2 \sigma^{2}\right),
$$

where ${ }_{1} F_{1}$ is the confluent hypergeometric function. This can be expressed in terms of Bessel functions (e.g., Talukdar \& Lawing 1991) as

$$
\langle\mu\rangle=\sigma \sqrt{\pi / 2} e^{-x / 2}\left[(1+x) I_{0}(x / 2)+x I_{1}(x / 2)\right],
$$

with $x=\mu^{2} / 2 \sigma^{2}$. The variance of the Rice distribution is given by

$$
\sigma_{\langle\mu\rangle}^{2}=2 \sigma^{2}+\mu^{2}-\langle\mu\rangle^{2} .
$$

To obtain an unbiased estimate for the proper motion, we solve Equation (10) for $\mu$ by setting $p=\langle\mu\rangle$. Equation (11) is then used to determine the uncertainty on the proper motion.

\section{ORCID iDs}

Toshiki Sato (1D https://orcid.org/0000-0001-9267-1693 Satoru Katsuda (1) https://orcid.org/0000-0002-1104-7205 Aya Bamba (iD https://orcid.org/0000-0003-0890-4920 John P. Hughes (i) https://orcid.org/0000-0002-8816-6800 Federico Fraschetti (i) https://orcid.org/0000-0002-5456-4771

\section{References}

Acciari, V. A., Aliu, E., Arlen, T., et al. 2010, ApJ, 714, 163

Ackermann, M., Ajello, M., Allafort, A., et al. 2013, Sci, 339, 807

Aharonian, F., Akhperjanian, A., Barrio, J., et al. 2001, A\&A, 370, 112

Albert, J., Aliu, E., Anderhub, H., et al. 2007, A\&A, 474, 937

Anderson, M. C., \& Rudnick, L. 1995, ApJ, 441, 307

Arnaud, K. A. 1996, in ASP Conf. Ser. 101, Astronomical Data Analysis Software and Systems V, ed. G. H. Jacoby \& J. Barnes (San Francisco, CA: ASP), 17

Bamba, A., Yamazaki, R., Yoshida, T., Terasawa, T., \& Koyama, K. 2005, ApJ, 621, 793

Bell, A. R. 1978, MNRAS, 182, 443

Berezhko, E. G., \& Ellison, D. C. 1999, ApJ, 526, 385

Berezhko, E. G., \& Völk, H. J. 2004, A\&A, 419, L27

Blandford, R., \& Eichler, D. 1987, PhR, 154, 1

Cash, W. 1979, ApJ, 228, 939

DeLaney, T., Rudnick, L., Fesen, R. A., et al. 2004, ApJ, 613, 343

DeLaney, T., Rudnick, L., Stage, M. D., et al. 2010, ApJ, 725, 2038

Eichler, D. 1984, ApJ, 277, 429

Ellison, D. C., Decourchelle, A., \& Ballet, J. 2005, A\&A, 429, 569

Eriksen, K. A., Hughes, J. P., Badenes, C., et al. 2011, ApJL, 728, L28

Farnebäck, G. 2003, in Image Analysis (Berlin: Springer), 363

Fesen, R. A., Hammell, M. C., Morse, J., et al. 2006, ApJ, 645, 283

Fruscione, A., McDowell, J. C., Allen, G. E., et al. 2006, Proc. SPIE, 6270 $62701 \mathrm{~V}$

Gotthelf, E. V., Koralesky, B., Rudnick, L., et al. 2001, ApJL, 552, L39 Grefenstette, B. W., Fryer, C. L., Harrison, F. A., et al. 2017, ApJ, 834, 19 Grefenstette, B. W., Harrison, F. A., Boggs, S. E., et al. 2014, Natur, 506, 339 Grefenstette, B. W., Reynolds, S. P., Harrison, F. A., et al. 2015, ApJ, 802, 15 Guberman, D., Cortina, J., de Oña Wilhelmi, E., et al. 2017, arXiv:1709.00280 Harrison, F. A., Craig, W. W., Christensen, F. E., et al. 2013, ApJ, 770, 103 Helder, E. A., \& Vink, J. 2008, ApJ, 686, 1094

Hester, J. J., Raymond, J. C., \& Blair, W. P. 1994, ApJ, 420, 721 
Hitomi Collaboration, Aharonian, F., Akamatsu, H., et al. 2016, Natur, 535, 117 Hitomi Collaboration, Aharonian, F., Akamatsu, H., et al. 2017, PASJ, in press (arXiv:1711.00240)

Holt, S. S., Gotthelf, E. V., Tsunemi, H., \& Negoro, H. 1994, PASJ, 46, L151

Hughes, J. P., Rakowski, C. E., Burrows, D. N., \& Slane, P. O. 2000, ApJL, 528, L109

Hwang, U., Holt, S. S., \& Petre, R. 2000, ApJL, 537, L119

Hwang, U., \& Laming, J. M. 2012, ApJ, 746, 130

Hwang, U., Laming, J. M., Badenes, C., et al. 2004, ApJL, 615, L117

Inoue, T., Yamazaki, R., Inutsuka, S.-I., \& Fukui, Y. 2012, ApJ, 744, 71

Jokipii, J. R. 1987, ApJ, 313, 842

Katsuda, S., Petre, R., Mori, K., et al. 2010, ApJ, 723, 383

Keohane, J. W., Rudnick, L., \& Anderson, M. C. 1996, ApJ, 466, 309

Kilpatrick, C. D., Bieging, J. H., \& Rieke, G. H. 2014, ApJ, 796, 144

Koyama, K., Petre, R., Gotthelf, E. V., et al. 1995, Natur, 378, 255

Laming, J. M. 2001, ApJ, 563, 828

Lazendic, J. S., Dewey, D., Schulz, N. S., \& Canizares, C. R. 2006, ApJ, 651, 250

Maeda, Y., Uchiyama, Y., Bamba, A., et al. 2009, PASJ, 61, 1217

Morse, J. A., Fesen, R. A., Chevalier, R. A., et al. 2004, ApJ, 614, 727

Parizot, E., Marcowith, A., Ballet, J., \& Gallant, Y. A. 2006, A\&A, 453, 387

Patnaude, D. J., \& Fesen, R. A. 2007, AJ, 133, 147

Patnaude, D. J., \& Fesen, R. A. 2009, ApJ, 697, 535

Patnaude, D. J., \& Fesen, R. A. 2014, ApJ, 789, 138
Patnaude, D. J., Vink, J., Laming, J. M., \& Fesen, R. A. 2011, ApJL, 729, L28 Reed, J. E., Hester, J. J., Fabian, A. C., \& Winkler, P. F. 1995, ApJ, 440, 706 Reynolds, S. P. 2008, ARA\&A, 46, 89

Sanders, J. S. 2006, MNRAS, 371, 829

Sano, H., Fukuda, T., Yoshiike, S., et al. 2015, ApJ, 799, 175

Sano, H., Sato, J., Horachi, H., et al. 2010, ApJ, 724, 59

Sano, H., Tanaka, T., Torii, K., et al. 2013, ApJ, 778, 59

Sato, T., \& Hughes, J. P. 2017a, ApJ, 840, 112

Sato, T., \& Hughes, J. P. 2017b, ApJ, 845, 167

Sato, T., Maeda, Y., Bamba, A., et al. 2017, ApJ, 836, 225

Serkowski, K. 1958, AcA, 8, 135

Stage, M. D., Allen, G. E., Houck, J. C., \& Davis, J. E. 2006, NatPh, 2, 614

Talukdar, K. K., \& Lawing, W. D. 1991, ASAJ, 89, 1193

Telezhinsky, I., Dwarkadas, V. V., \& Pohl, M. 2012, A\&A, 541, A153

Telezhinsky, I., Dwarkadas, V. V., \& Pohl, M. 2013, A\&A, 552, A102

Uchiyama, Y., \& Aharonian, F. A. 2008, ApJL, 677, L105

Uchiyama, Y., Aharonian, F. A., Tanaka, T., Takahashi, T., \& Maeda, Y. 2007, Natur, 449, 576

Vink, J., \& Laming, J. M. 2003, ApJ, 584, 758

Vink, J., \& Yamazaki, R. 2014, ApJ, 780, 125

Williams, B. J., Coyle, N. M., Yamaguchi, H., et al. 2017, ApJ, 842, 28

Willingale, R., Bleeker, J. A. M., van der Heyden, K. J., Kaastra, J. S., \& Vink, J. 2002, A\&A, 381, 1039 